Running head: Children's predictions about illness

Paper published at Child Development, doi: 10.1111/cdev.13655

Young children's ability to make predictions about novel illnesses

Jasmine M. DeJesus, ${ }^{1}$ Shruthi Venkatesh, ${ }^{1} \&$ Katherine D. Kinzler ${ }^{2}$

${ }^{1}$ Department of Psychology, UNC Greensboro

${ }^{2}$ Department of Psychology, University of Chicago

Address for correspondence:

Jasmine M. DeJesus

Department of Psychology

321 McIver St.

UNC Greensboro

Greensboro, NC 27412

Email: jmdejes2@uncg.edu

ORCID: 0000-0002-0981-2481

Acknowledgements. This research was supported by NICHD grant R01 HD070890 to K.D.K. and NSF Graduate Research Fellowship (DGE-1144082) to J.M.D. Thanks to Molly Gibian, Lucy Tindel, Sasha Diaz, Madison Ellis, Déja Bryden, Samantha Alvarado, Yazdan Shah, Hannah McEachern, Todd Ross, Jaylan Graham, and Stanysha Lowery for assistance in recruitment and data collection, to the Parent Researcher Collaborative (including Elizabeth Bonawitz, Hyowon Gweon, Julian Jara Ettinger, Candice Mills, Laura Schulz, and Mark Sheskin), and to Shaylene Nancekivell and Isobel Heck for helpful conversation about this project. We especially thank the families who participated in this study during the COVID-19 pandemic. 


\begin{abstract}
Understanding disease transmission is a complex problem highlighted by the COVID-19 pandemic. These studies test whether 3- to 6-year-old children in the United States use information about social interactions to predict disease transmission. Before and during COVID19, children predicted illness would spread through close interactions. Older children outperformed younger children, with no associations between task performance and pandemic experience. Children did not predict that being hungry or tired would similarly spread through close interactions. Participants include 196 3 -6-year-olds (53\% girls, 47\% boys; 68\% White, 9\% Black, 7\% Asian, 6\% Hispanic or Latinx), with medium-sized effects $\left(d=0.6, \eta_{p}{ }^{2}=0.3\right)$. These findings suggest that thinking about social interaction supports young children's predictions about illness, with noted limitations regarding children's real-world avoidance of diseasespreading behaviors.
\end{abstract}

Keywords: Contagion; cognitive development; illness; intuitive theories; COVID-19 


\section{Young children's ability to make predictions about novel illnesses}

According to the Center for Disease Control, adults suffer from an average of two to three colds per year, and children are even more susceptible to contracting colds and other contagious illnesses (Centers for Disease Control and Prevention, 2016a). Prior to the COVID-19 pandemic, thousands of children were hospitalized annually from influenza and related complications, more than any other vaccine-preventable illness (Centers for Disease Control and Prevention, 2016b; Kloepfer et al., 2014; Rolfes et al., 2016). As such, understanding how illness spreads is an interesting developmental problem with important public health consequences. Notably, understanding and avoiding contaminants appears to be difficult for young children (Fallon et al., 1984; Rozin \& Fallon, 1987; Solomon \& Cassimatis, 1999; Stevenson et al., 2010). Preschool-aged children are willing to touch and ingest things that adults and older children would avoid - dirty objects, food that appears to be sneezed on, or food that contains a contaminant (e.g., DeJesus et al., 2015; Fallon et al., 1984; Stevenson et al., 2010) and they have difficulty making consistent predictions about who might get sick and what people could do to avoid contaminants (Legare et al., 2009). It is not until the early elementary school years that children avoid interacting with a person who showed symptoms of illness (Blacker \& LoBue, 2016), and children's drawings of sick people increase in complexity (i.e., the types of graphic and contextual cues employed) across this same period (Bonoti et al., 2019).

One reason why illness may be so difficult to understand from an early age is that the bacteria and viruses that cause illness are not visible to the naked eye, so children cannot directly track them. Rozin and colleagues argue that contamination is hard for young children to understand because it requires them to consider that appearances may differ from reality (i.e., what looks good to eat may not be if it came in contact with a pathogen or toxin) and that small 
particles like germs and poisons exist and operate even though we cannot see them (e.g., Rozin \& Fallon, 1987). Although children can successfully infer causes from their own observations in multiple domains (e.g., Gopnik, 2012; Gopnik et al., 2004; Xu \& Tenenbaum, 2007), the underlying causes of illness are themselves often opaque and not perfectly deterministic. As an illustration, in a study examining the link between stress and disease susceptibility in adults, all participants were directly exposed to the common cold virus ("participants were given nasal drops containing a low infectious dose of one of two types of rhinovirus," p. 415), yet only $40 \%$ ultimately developed cold symptoms (Cohen et al., 1998). The COVID-19 pandemic has highlighted both the importance and difficulty of understanding disease transmission, especially for illnesses that can be transmitted by asymptomatic people. More than half of COVID-19 cases are spread by people without symptoms (Johansson et al., 2021). Although children are much less likely than adults to experience severe outcomes from the illness, they nonetheless are susceptible to the virus and can transmit it to others (American Academy of Pediatrics, 2020). Therefore, concerns exist about the safety of children in school and daycare settings, the safety of people who interact with children (such as their parents, grandparents, and teachers), and children's ability to understand how COVID-19 can be transmitted.

Though children's direct observations may not always give them reliable insights into the causes of illness, their naïve biological reasoning may provide an important entry point prior to acquiring a thorough understanding of the mechanisms of disease transmission. Even young children are able to make judgments about living vs. nonliving things and prioritize internal features as key to causal processes (Gelman, 2003; Gelman \& Wellman, 1991; Margett-Jordan et al., 2017; Sobel et al., 2007). The existence of these early biological theories may explain why even young children appear to reason effectively about illness in some tasks. For instance, 
preschool-aged children demonstrate knowledge that the presence or absence of germs is relevant for illness (Kalish, 1996a; Solomon \& Cassimatis, 1999), they are capable of invoking germs and biological concepts to explain why someone got sick (Legare et al., 2009), and by 4 years of age children understand that the causes and cures of illness unfold over time (Raman \& Gelman, 2007). When provided with competing explanations about why someone got sick, they are more likely to select biological explanations for illness (e.g., explanations that invoke germs or bodily fluids) than explanations that rely on desires or punishment for bad behavior (Hatano \& Inagaki, 2013; Kalish, 1997; Siegal, 1988; Slaughter \& Lyons, 2003; Springer \& Ruckel, 1992). By 7 years of age, children use information about the type of disease (contagious vs. not contagious), type of contact (varying levels of physical contact), and frequency of contact to make predictions about the risk of contagion from a sick person (Muñoz Marco et al., 2017). Perhaps children can derive a simple strategy from these early conceptual foundations to inform their predictions about illness, which would not require a detailed understanding of germs, invisible particles, or causality.

The present research examines children's understanding of close interactions as a potentially important cue to disease transmission. In Experiment 1, 3- to 6-year-old children were told about two characters who had a playdate, even though one character was sick with a novel illness. We then examined whether children would use information about how closely characters interacted (e.g., near each other and frequently touching vs. far away and not touching) to predict whether one character would get sick from the other. We chose to assess children's predictions specifically because prior studies have shown that 3-and 4-year-old children are already able to understand why someone got sick after learning about that outcome, but struggled to make reliable predictions (Legare et al., 2009), therefore we aimed to examine 
whether information about close interactions would improve the predictions of our youngest participants. We hypothesized that children would use information about close interactions to inform their predictions about illness and that this ability would increase with age. In Experiment 2, we tested whether children would use similar information about interactions to make predictions about other internal states (e.g., being hungry or tired) to examine whether children's consideration of close interactions is unique to illness.

Experiment 1 and 2 were conducted before the COVID-19 pandemic. In Experiments 3 and 4, we replicated and extended the methods of Experiment 1 in a videoconference format to examine potential effects of the pandemic on children's reasoning about disease transmission. The COVID-19 pandemic is an especially interesting context in which to consider these questions, as children have been learning about a real novel illness, have been restricted from interacting with others, and might be having more explicit conversations about disease transmission at home.

\section{Experiment 1: Children's Predictions About Disease Transmission}

In Experiment 1, we examined whether 3- to 6-year-old children consider close interactions between two people as a cue to the likelihood of disease transmission. Children heard stories about two children who played together that referenced different levels of contact. One child was sick and participants were asked to predict whether or not the other child would get sick after the playdate. Data were collected before the COVID-19 pandemic (2013).

\section{Method}

Participants

Participants included 48 three- to six-year-old children (22 boys, 26 girls; $M=4.96$ years, range $=3.03-6.85$ years) from the Midwestern United States. Children were recruited from two 
age groups: $3-4$-year-olds $(N=24)$ and 5-6-year-olds $(N=24)$. This sample size was based on prior studies of preschoolers' predictions about illness (Legare et al., 2009). This age range was selected to examine the emergence of this ability, as studies of naïve biological thinking and children's ability to avoid contamination demonstrate an emergence around the age of 5 (e.g., Blacker \& LoBue, 2016; DeJesus et al., 2015; Hatano \& Inagaki, 2013), and very few find any evidence of successful predictions about disease or biological concepts among 3- and 4-year-old children (e.g., Legare et al., 2009). We planned to recruit equal numbers of 3-4-year-olds and 56-year-olds. In terms of race, 35 children were White, 6 were Black or African American, 2 were Asian or Asian American, and 5 were biracial or other according to parental report (0 American Indian or Alaska Native; 0 Native Hawaiian or Other Pacific Islander). In terms of ethnicity, 1 was Hispanic or Latinx and 47 were not Hispanic or Latinx. Children participated in only one experiment in the presented research (e.g., a child who participated in Experiment 1 could not have participated in Experiments 2-4).

\section{Materials and Procedure}

Participants viewed cartoon images of children on a laptop computer (see Figure 1). The experimenter stated that the top character was sick with a novel disease; a red box appeared around the sick character's face. The experimenter then described the nature of the interaction between characters during a playdate (low, medium, or high interaction; see Appendix). In high interaction trials, the two characters "play right next to each other, and touch each other a lot." In medium interaction trials, the two characters "play nearby each other, and only touch each other a little bit." In low interaction trials, the two characters "play across the room for each other, and don't touch each other at all." No visual information was presented to provide clues 
or reminders about the level of contact during the trial to use the same stimuli across trials and experiments.

Figure 1 goes here

Children were then asked to predict whether the bottom character would also get sick with the novel illness after their playdate and how sure they were about their choice. For instance, if children responded that the character would get sick, they were then asked, "Is s/he definitely going to get sick or is s/he maybe going to get sick?" Children saw two exemplars of each type of contact (one male, one female).

\section{Design}

The order in which different interaction types were presented was counterbalanced within and across participants. Characters were gender-matched in case children might hold different expectations about play behavior or disease transmission for gender-matched vs. cross-gender pairs. The gender of each pair alternated; half of children saw a male pair first and half saw a female pair first. Across all experiments, vignettes were designed to sound like real-life situations, while avoiding diseases that children might know about or have experienced directly (such as a cold or flu), and avoid introducing impossible situations (e.g., characters who played far away did not touch each other).

\section{Scoring and Analysis}

Children's responses to the sick question and the certainty question were combined to form a four-point scale. "Definitely no" was scored as 0, "maybe no" was scored as 1, "maybe yes" was scored as 2, and "definitely yes" was scored as 3. Children's score for the two exemplars of each interaction type were averaged to provide a mean score for each level of contact (low, medium, high). Children's responses were analyzed using a repeated measures 
ANOVA, with interaction type entered as a within-subjects variable and age group (3-4-years, 5-6-years) entered as a between-subjects variable. Resulting pairwise comparisons are provided to test for differences between contact types (low vs. medium, medium vs. high, low vs. high); paired-sample t-tests were considered significant at $p=.017$ to account multiple comparisons $(p$ $=.05$ divided by $3=.017)$. Cohen's $d$ and $\eta_{p}{ }^{2}$ are presented as measures of effect size. These are confirmatory analyses designed to test the prediction that children would use interaction type as a guide to disease transmission.

Deidentified data, analysis code, and study materials are available on the Open Science Framework (OSF, https://osf.io/s4ubt).

\section{Results}

Children used information about how characters interacted when predicting whether a story character might get sick. A repeated measures ANOVA revealed a significant effect of interaction type, $F(2,92)=33.0, p<.001, \eta_{p}{ }^{2}=0.42$. Children were more likely to predict that characters would get sick for high interaction trials $(M=1.98,95 \% C I=1.74,2.22)$ than low interaction trials $(M=0.99,95 \% C I=0.80,1.18), t(47)=6.21, p<.001, d=0.90$, for high interaction trials than medium interaction trials $(M=1.51,95 \% C I=1.35,1.67), t(47)=4.39, p$ $<.001, d=0.63$, and for medium interaction trials than low interaction trials, $t(47)=4.52, p<$ $.001, d=0.65$.

There was no main effect of age group, $F(1,46)=0.17, p=.678, \eta_{p}{ }^{2}<0.01$. However there was a significant interaction between interaction type and age group, $F(2,92)=6.75, p=$ $.002, \eta_{p}{ }^{2}=0.13$. Further inspection of the data suggests that the effect of interaction was more robust among 5- and 6-year-olds than 3- and 4-year-olds, but the effect nonetheless was observed among the 3-4-year-old group: Separate repeated measures ANOVAs for each age group 
revealed a significant effect of interaction type $\left(3-4: F(2,46)=5.54, p=.007, \eta_{p}{ }^{2}=0.19 ; 5-6\right.$ : $\left.F(2,46)=31.4, p<.001, \eta_{p}{ }^{2}=0.58\right)$. An independent-samples $t$-test comparing the difference between high and low interaction by age group reveals that older children (5-6-year-olds; $M=$ $1.44,95 \% C I=0.98,1.90)$ demonstrated a bigger difference between high and low interaction than younger children (3-4-year-olds; $M=0.54,95 \% C I=0.14,0.94), t(45.11)=3.05, p=.004$, $d=0.88$.

\section{Discussion}

Despite past findings that young children have difficulty making predictions about illness (Legare et al., 2009), reasoning about germs and illness (Carey, 1985; Kalish, 1996b; Solomon \& Cassimatis, 1999), and physically avoiding contaminants (Blacker \& LoBue, 2016; DeJesus et al., 2015), the results of Experiment 1 suggest that young children can use information about close interactions to inform their thinking about the spread of disease. When asked to predict whether a story character might get sick, children across ages were more likely to say that characters were more likely to get sick after closely interacting with a sick person. Although past scholarship suggests that reasoning about invisible entities and disease transmission can be particularly difficult for young children (Au et al., 1993; Blacker \& LoBue, 2016; Kalish, 1998; Rozin \& Fallon, 1987; Stevenson et al., 2010), the children tested here successfully used information about close interactions to make predictions about illness. Though this effect was particularly robust among 5- and 6-year-old participants (consistent with the developmental trends described previously), we nonetheless observed a significant effect among 3- and 4-yearolds. In line with children's real-life experiences with germs, no visual information was provided to support children's predictions, yet even our youngest participants reasoned that contact between two people was relevant for disease transmission. 
Is children's reasoning about the consequences of contact unique to illness? Children may simply infer that people who interact with each other are more likely to take on each other's internal states. If so, then children's reasoning about contact in Experiment 1 might not reflect any reasoning about illness, but instead might suggest that children assume that people who are near each other are likely to be similar. Experiment 2 tests this possibility by presenting children with the same stories as in Experiment 1, but with illness replaced by another internal state (being hungry or tired).

\section{Experiment 2: Children's Predictions About Other Internal States}

In Experiment 2, we tested whether children's reasoning about close interactions in Experiment 1 is specific to illness. We presented children with a version of the vignettes from Experiment 1 but asked whether children thought one character would become hungry or tired after interacting with a character who was hungry or tired. Data were collected before the COVID-19 pandemic (2013-2014).

\section{Method}

\section{Participants}

Participants included 48 three- to six-year-old children (25 boys, 23 girls; $M=4.91$ years, range $=3.03-6.84$ years) from the Midwestern United States. Children were recruited from two age groups: 3-4-year-olds $(N=24)$ and 5-6-year-olds $(N=24)$. In terms of race, 31 children were White, 8 were Black or African American, 1 was Asian or Asian American, and 7 were biracial or other according to parental report (0 American Indian or Alaska Native; 0 Native Hawaiian or Other Pacific Islander; 1 parent did not report the child's race). In terms of ethnicity, 5 were Hispanic or Latinx and 43 were not Hispanic or Latinx.

\section{Materials, Procedure, and Design}


The materials and procedure were identical to Experiment 1, with the exception that children were told that the top character was either hungry or tired (rather than sick) and asked whether the bottom character would become hungry or tired following the characters' playdate (see Appendix). As in Experiment 1, the order of vignettes was counterbalanced within and across participants. Pairs were gender-matched and the gender of each pair alternated; half of children saw a male pair first and half saw a female pair first. Children saw one exemplar of each internal state (hungry, tired) at each contact level (low, medium, high) for a total of six trials.

\section{Scoring and Analysis}

Children's responses were scored on a 0-3 scale as in the prior experiment. The same analyses were conducted as in Experiment 1 . These are confirmatory analyses designed to test the prediction that children would not use interaction type as a guide to internal states besides illnesses (i.e., feeling hungry or tired).

\section{Results}

We found no significant effect of interaction type in Experiment 2, $F(2,92)=2.32, p=$ .104 (low interaction trials: $M=1.63,95 \% C I=1.45,1.81$; medium interaction trials: $M=1.43$, 95\% $C I=1.24,1.63$; high interaction trials: $M=1.46,95 \% C I=1.27,1.65)$. We also did not observe an effect of age group, $F(1,46)=3.25, p=.078$, or interaction between age group and interaction, $F(2,92)=0.38, p=.683$. These results suggest that children do not believe that all internal states are spread by interacting with other people.

\section{Discussion}

The results of Experiment 2 provide evidence that children do not expect close interactions between people to transmit any internal state. Unlike predictions about illness (as 
depicted in Experiments 1), children tested in Experiment 2 did not use the closeness of interactions to predict whether a character would later become hungry or tired.

After the onset of COVID-19, we sought to replicate Experiment 1 to examine the extent to which the pandemic altered children's predictions about novel illnesses. Coincidentally, the pandemic includes several of the features that we highlighted in our study vignettes - COVID-19 is a novel illness that is transmitted by closely interacting with other people. Children's early conceptual knowledge may be sufficient for them to make reliable predictions about disease transmission. Given that they are already successful at making predictions in Experiment 1, even a highly salient and relevant event may not substantially alter their predictions. Yet, there are many reasons to expect that children's reasoning could be affected by the pandemic. It may be even more obvious to children that interaction promotes disease transmission, and children may appreciate the link between contact and disease transmission at an earlier age than they would have in ordinary circumstances. Parents could be discussing disease transmission much more at home and this could serve as an early intervention on causal mechanisms about illness (see Au et al., 2008, for related findings among older children). Alternatively, children could be overwhelmed with disease messaging and view any interaction (even socially distanced ones) as likely to cause someone to get sick.

\section{Experiment 3: Children's Predictions About Illness During COVID-19}

To examine the potential effects of the pandemic on children's thinking about disease transmission, we replicated Experiment 1 in an online videoconference format (data collected in 2020-21). Parents also participated in a structured interview to better understand how they had been discussing disease transmission at home and how the pandemic has affected their families.

\section{Method}




\section{Participants}

Participants included 48 three- to six-year-old children (22 boys, 26 girls; $M=5.10$ years, range $=3.06-6.88$ years) from a variety of locations, primarily the Southeastern and Midwestern United States (recruited from local volunteer family databases, the Children Helping Science platform, childrenhelpingscience.com, and social media advertisements). Children were recruited from two age groups: 3-4-year-olds $(N=24)$ and 5-6-year-olds $(N=24)$. In terms of race, 33 children were White, 1 was Black or African American, 5 were Asian or Asian American, and 6 were biracial or other according to parental report (0 American Indian or Alaska Native; 0 Native Hawaiian or Other Pacific Islander; 3 parents did not report their child's race). In terms of ethnicity, 2 were Hispanic or Latinx and 43 were not Hispanic or Latinx (3 parents did not report their child's ethnicity).

An additional 6 children were excluded because they did not complete the study $(N=2)$, their parents or sibling interfered during the study (by suggesting an answer or translating for the child, $N=3)$, or they were younger than 3 when they completed the study $(N=1)$.

\section{Materials, Procedure, and Design}

The materials and procedure were identical to Experiment 1, with the exception that the study was conducted online using videoconferencing software (Webex and Zoom). Children were shown the same images as in Experiment 1 through screen sharing and an experimenter read the same script text to the child. To ensure that children could see the experimenter and the study images, participants first completed a two-part calibration test. After sharing their screen, the experimenter first made a thumbs-up sign and asked children if they could do the same. Then the experimenter showed the child a picture of a blue star and a red circle (see Figure 2) and asked children to name the color of each shape. For any child who had difficulty naming the 
colors (e.g., one parent reported that their child was colorblind), children were asked which shape was bigger. All children passed the thumbs-up check and at least one of the image checks (shape color or size). Parents were sometimes nearby to help set up the video conference but were asked not to help children make their guesses and told that any answers would be interesting for us to know.

Figure 2 goes here

Children then completed the task described in Experiment 1. After the 6 trials of this task, the experimenter asked an open-ended follow-up question: "Is there anything else you want to tell me about getting sick?" Following the child's portion of the study, parents were asked a series of questions about their discussions about COVID-19, germs, and illness and their experience during the pandemic (see Appendix for full text of questions and Experiment 4 for additional descriptive data and associations with children's reasoning about disease transmission).

\section{Scoring and Analysis}

Children's responses were scored on a 0-3 scale as in prior experiments and were analyzed in the same manner as Experiment 1 . These are confirmatory analyses designed to test the prediction that children would use interaction type as a guide to disease transmission.

We provide descriptive data from the open-ended questions and parent interview. At the end of Experiment 4, we provide an exploratory analysis of potential associations between these variables and their performance in this task in a larger sample of children tested during the pandemic.

\section{Results}


As in Experiment 1, children used information about contact when predicting whether a story character might get sick. A repeated measures ANOVA revealed a significant effect of interaction type, $F(2,92)=17.69, p<.001, \eta_{p}{ }^{2}=0.28$. Children were more likely to predict that characters would get sick for high interaction trials $(M=2.93,95 \% C I=1.76,2.30)$ than low interaction trials $(M=1.09,95 \% C I=0.83,1.35), t(47)=4.69, p<.001, d=0.68$, for high interaction trials than medium interaction trials $(M=1.68,95 \% C I=1.47,1.89), t(47)=2.66, p$ $=.011, d=0.38$, and for medium interaction trials than low interaction trials, $t(47)=3.89, p<$ $.001, d=0.56$.

There was no main effect of age group, $F(1,46)=1.22, p=.275$. However there was a significant interaction between interaction type and age group, $F(2,92)=3.61, p=.031, \eta_{p}{ }^{2}=$ 0.07. Separate repeated measures ANOVAs for each age group revealed a significant effect of interaction type for 5- and 6-year-olds, $F(2,46)=21.12, p<.001, \eta_{p}{ }^{2}=0.48$, but not 3- and 4year-olds, $F(2,46)=2.79, p=.072, \eta_{p}^{2}=0.11$.

\section{Comparing Across Experiments 1 and 3}

Comparing across Experiment 1 (before COVID, in person) and Experiment 3 (during COVID, by video conference), we observed a significant effect of interaction type, $F(2,184)=$ 46.52, $p<.001, \eta_{p}^{2}=0.34$, and a significant interaction between interaction type and age group, $F(2,184)=9.40, p<.001, \eta_{p}{ }^{2}=0.09$. No other main effects or interactions (including experiment, $p=.298$ ) were significant, $p \mathrm{~s}>.268$. For the effect of interaction type, children were more likely to predict that characters would get sick for high interaction trials $(M=2.01,95 \% C I$ $=1.83,2.18)$ than low interaction trials $(M=1.04,95 \% C I=0.88,1.20), t(95)=7.57, p<.001, d$ $=0.77$, for high interaction trials than medium interaction trials $(M=1.59,95 \% C I=1.46,1.73)$, 
$t(95)=4.84, p<.001, d=0.49$, and for medium interaction trials than low interaction trials, $t(95)$ $=5.87, p<.001, d=0.60$.

As in Experiment 1, further inspection of the data suggests that the effect of interaction type was more robust among 5- and 6-year-olds than 3- and 4-year-olds, but the effect nonetheless was significant among the 3-4-year-old group. Separate repeated measures ANOVAs for each age group revealed a significant effect of interaction type $(3-4: F(2,92)=$ 7.07, $\left.p=.001, \eta_{p}{ }^{2}=0.13 ; 5-6: F(2,92)=51.05, p<.001, \eta_{p}{ }^{2}=0.53\right)$. No effects of experiment $(p s>.177)$ or interactions $(p s>.779)$ were observed in either age group. An independentsamples $t$-test comparing the difference between high and low interaction by age group reveals that older children $(5-6$-year-olds; $M=1.40,95 \% C I=1.06,1.73)$ demonstrated a bigger difference between high and low interaction than younger children (3-4-year-olds; $M=0.53$, $95 \% C I=1.82,0.88), t(93.82)=3.61, p<.001, d=0.74$.

\section{Open-ended Child Responses}

Few children provided open-ended responses when asked, "Is there anything else you want to tell me about getting sick?" Eleven children provided some response; of those, 3 were at least partially prompted by a parent. Responses included discussions of general illness symptoms (e.g., "tummy hurt", "throwing up"), referencing a sibling who had been sick, or specific reference to COVID (e.g., "6 feet away, wearing a mask, and that's all", "You have to go to the hospital and then you will get COVID”, "big bad virus"). Interestingly, only one child commented on the study vignettes themselves: "When you get sick you definitely shouldn't go to a playdate with your friend."

\section{Parent Interview}


See Table 1 for parent interview responses by experiment. The majority of parents mentioned talking to their participating child about germs daily $(27$, or $56 \%)$, had increased the amount they talked about germs since the pandemic started (40,83\%), and had taught their participating child a new germ-related behavior $(35,73 \%)$. Some children participated in the study before mask wearing was widely recommended or when locations frequented by children (daycare, school, public parks) were closed, so wearing masks or keeping distance from people outside the home might not have been relevant for some families at the time they were tested. In terms of the time since children had been in school or daycare, 3 children had not yet started attending school or daycare at the time they participated in the study. Of the remaining 45 children, they ranged greatly in the amount of time since they had been to school in person, ranging from 0 days (meaning they had been to school in person the day they completed the study) to 330 days (mean $=124$ days). The majority of children $(67 \%, 32$ out of 48$)$ had not attended school or daycare since the pandemic started; 33\% (16 out of 48) had returned to school or daycare in person by the time they participated in the study. We observed a similarly wide range of responses for the number of days since children had last had a playdate: 0 to 311 days; mean $=56$ days. Playdates included socially distanced or masked interactions, interactions outside, and interactions with families in the participating family's "bubble."

Table 1 goes here

\section{Discussion}

Overall, children's predictions about illness in Experiment 3 (conducted during the COVID-19 pandemic) largely mirror children's predictions prior to the pandemic. Children considered close interactions as more likely to transmit illness, particularly among older children (5- and 6-year-olds) in our sample. We did not observe differences between Experiment 1 and 3, 
suggesting that neither the move to testing by video conference (rather than in person) nor children's experience during the pandemic largely impacted their performance in this task. Nonetheless, with just one sample of children tested during the pandemic, we are hesitant to draw a strong conclusion about their experience during COVID-19 (including potential differences in their return to school or parent talk about disease transmission).

To further understand the potential impact of the pandemic on children's thinking about disease transmission, we conducted an additional experiment during COVID-19 to replicate and extend these findings. In addition, with a larger sample we had more power to examine individual or contextual differences that might be related to children's thinking about disease transmission. Given that data collection for these two studies spanned 11 months, children's thinking could vary based on the stage of the pandemic they were in or differences in their experience at home or school.

\section{Experiment 4: Extending Children's Predictions About Illness During COVID-19}

Experiment 4 sought to replicate and extend the findings of Experiment 3. In addition to providing a second sample of participants tested during the pandemic, Experiment 4 examined whether children primarily use information about touch (i.e., touching a lot vs. not at all) compared to proximity (i.e., whether characters are close or far from each other) when making predictions about disease transmission. Children heard an amended version of the medium interaction trial (previously involving playing nearby each other, and touching each other a little bit) designed to share one feature each of the high and low contact statements - in this vignette, children were described as playing right next to each other (similar to the high contact trial) but not touching (similar to the low contact trial), allowing us to assess whether one or both factors contribute to children's thinking about disease transmission. The rest of the experiment was 
identical to Experiment 3, including testing by video conference, the same high and low interaction trials, and the same parent interview.

\section{Method}

\section{Participants}

Participants included 52 three- to six-year-old children (24 boys, 28 girls; $M=5.04$ years, range $=3.10-6.58$ years) from a variety of locations, primarily the Southeastern and Midwestern United States (recruited from local volunteer family databases, Children Helping Science, and social media advertisements). Children were recruited from two age groups: 3-4year-olds $(N=25)$ and 5-6-year-olds $(N=27)$. In terms of race, 35 children were White, 2 were Black or African American, and 6 were Asian or Asian American according to parental report (0 American Indian or Alaska Native; 0 Native Hawaiian or Other Pacific Islander; 0 biracial; 0 other; 9 parents did not report their child's race). In terms of ethnicity, 4 were Hispanic or Latinx and 42 were not Hispanic or Latinx (6 parents did not report their child's ethnicity).

Two additional children were excluded because they did not wish to complete the study. Participants completed the study during the COVID-19 pandemic (2020-2021).

\section{Materials, Procedure, and Design}

The materials and procedure were identical to Experiment 3: Children completed the task by video conference (with checks to ensure they could see the experimenter and the shared screen) and were presented with examples of high, medium, and low contact playdate interactions between two children, one of whom was sick with a novel illness. There was one difference between Experiments 3 and 4: In Experiment 4, the middle level of contact ("play nearby each other and only touch each other a little bit") was replaced with a close proximity-no contact vignette ("play right next to each other and don't touch each other at all," see Appendix 
for full vignettes). Scoring and analyses were conducted in the same manner as prior experiments to conduct confirmatory analyses designed to test the prediction that children would use interaction type as a guide to disease transmission. In addition, we provide exploratory analyses to examine individual differences in children's experience of the pandemic (e.g., whether children who had experienced the pandemic for a longer period of time or whose parents talked about disease transmission more at home would better understand the link between close interactions and disease transmission).

\section{Results}

A repeated measures ANOVA revealed a significant effect of interaction type, $F(2,100)$ $=17.60, p<.001, \eta_{p}{ }^{2}=0.26$. Children were more likely to predict that characters would get sick for high interaction trials $(M=1.80,95 \% C I=1.53,2.07)$ than low interaction trials $(M=1.12$, $95 \% C I=0.92,1.31), t(51)=4.85, p<.001, d=0.67$, and for high interaction trials than medium interaction trials $(M=1.31,95 \% C I=1.09,1.52), t(51)=4.17, p<.001, d=0.58$, but did not differ between medium trials and low interaction trials, $t(51)=1.72, p=.091, d=0.23$.

There was no main effect of age group, $F(1,50)=2.40, p=.128, \eta_{p}{ }^{2}=0.05$. However there was a significant interaction between interaction type and age group, $F(2,100)=8.79, p<$ $.001, \eta_{p}{ }^{2}=0.15$. Separate repeated measures ANOVAs for each age group revealed a significant effect of interaction type for 5- and 6-year-olds, $F(2,52)=18.87, p<.001, \eta_{p}{ }^{2}=0.42$, but not 3and 4-year-olds, $F(2,48)=1.74, p=.187, \eta_{p}{ }^{2}=0.07$. Five- and 6-year-olds were more likely to predict that characters would get sick for high interaction trials $(M=2.17,95 \% C I=1.80,2.53)$ than low interaction trials $(M=1.02,95 \% C I=0.75,1.28), t(26)=5.11, p<.001, d=0.98$, and for high interaction trials than medium interaction trials $(M=1.43,95 \% C I=1.12,1.73), t(26)=$ 
4.26, $p<.001, d=0.82$, but did not differ between medium trials and low interaction trials after correction for multiple comparisons (criterion: $p=.017$ ), $t(26)=2.48, p=.020, d=0.48$.

We did not observe differences between Experiments 3 and 4: We observed no main effect of experiment, $F(1,96)=2.83, p=.096$, or interactions between experiment and other factors (Experiment $\mathrm{x}$ Interaction type: $F(2,192)=2.21, p=.113$; Experiment $\mathrm{x}$ Interaction type x Age group: $F(2,192)=0.13, p=.874)$. Similar to prior experiments, we observed an effect of interaction type, $F(2,192)=33.91, p<.001, \eta_{p}{ }^{2}=0.26$, and an interaction between age group and interaction type, $F(2,192)=10.90, p<.001, \eta_{p}{ }^{2}=0.10$, but no main effect of age group, $F(1,96)=3.53, p=.063$.

\section{Individual Differences in Children's Pandemic Experiences}

Effects of pandemic timing. To examine potential effects of pandemic stage, we categorized participants into three groups: pre-COVID $(N=48$, Experiment 1$)$, early COVID (children tested in 2020, $N=49$ ), and late COVID (children tested in 2021, $N=51$ ). These categories were selected by considering having as equal groups as possible without dividing children tested on the same day. We ran a two-way ANOVA to examine whether children's responses differed by pandemic timing. Specifically, we analyzed the difference in children's responses in high and low interaction trials as an index of the strength of children's use of interaction type. We observed a significant effect of age group, $F(1,142)=25.13, p<.001, \eta_{p}{ }^{2}=$ 0.15 , but no significant effect of timing, $F(2,142)=0.50, p=.607$, or interaction between timing and age group $F(2,142)=0.48, p=.622$ (see Figure 3 ). Replicating the interaction between age group and interaction type reported in individual experiments, across experiments older children demonstrated a bigger difference between high and low interaction trials $(M=1.31,95 \% C I=$ $1.04,1.57)$ compared to younger children $(M=0.41,95 \% C I=0.17,0.65)$. 
Figure 3 goes here

Despite the potential increase in salience of getting sick due to COVID-19, children tested before $(M=2.82$ out of a maximum of $6,95 \% C I=2.33,3.30)$ and during the pandemic (early: $M=2.90,95 \% C I=2.44,3.36$; late: $M=2.71,95 \% C I=2.15,3.27$ ) did not differ in the number of times children responded yes, that a character would get sick, $F(2,145)=0.15, p=$ .863.

Although we did not observe an effect of pandemic timing, the frequentist approach employed cannot differentiate between a null result in an ANOVA and the possibility that there is truly no difference in children's responses based on pandemic timing. To differentiate between these possibilities, we performed a Bayesian ANOVA with child age group (3-4 vs. 5-6), pandemic timing (pre-, early, and late COVID), and their interaction as effects on the high-low interaction trial difference. Compared to an intercept-only model, a model with age group had the highest Bayes factor $(9,121.47)$, meaning that a model with age group had roughly 9,000 times more evidence for the model than an intercept-only model (a very strong effect; Jarosz \& Wiley, 2014). In contrast, a model with timing only had a Bayes factor of 0.10. Models that including both age group and timing (Bayes factor $=1,043.46$ ) and age group, timing, and their interaction $($ Bayes factor $=190.47)$ also had some evidence in their favor, but the model with age group only was substantially better (compared to age group and timing: 8.74; compared to age group, timing, and their interaction: 47.89).

To provide a more fine-grained examination of children's performance in the task based on exactly when in the pandemic they were tested, we calculated a correlation between children's high-low difference score and the number of days between the date of test and March 11, 2020 (the day the World Health Organization declared COVID-19 to be a global pandemic, 
mean $=274$ days, $95 \% C I=256,291$ days). No significant association was observed, $r(101)=$ $0.04, p=.722$.

Individual differences in pandemic experience (Experiments 3 and 4). Parents whose children participated in the study during the pandemic $(N=100)$ were asked to complete a structured interview about their family's experiences during the pandemic (one parent declined to complete the interview). Descriptive statistics of parents' responses are presented in Table 1. The majority of parents discussed germs daily, increased their discussion of germs since COVID started, and had taught their child a new germ-related behavior. Most children had not yet returned to school or daycare in person at the time they participated in the study.

Nonetheless, children's experiences of the pandemic varied widely. Some children had been to school in person on the day they participated in the study. Although most children $(N=$ 74) did not provide an illness-related response to the open-ended question at the end of the session, 26 children spontaneously mentioned something about disease transmission, including naming illness symptoms, their own or their sibling's experience being sick, or something related to COVID-19 (as described in Experiment 3). Therefore, we next investigated whether contextual parental or environmental factors during the pandemic might be related to children's performance in Experiments 3-4. We performed a linear regression using parents' responses during the interview and whether children provided an illness-relevant open-ended response as predictors of the high-low difference score. Specifically, we included whether children provided an illness-related open-ended response $($ yes $=1$, no $=0$ ), parent germ talk frequency (daily $=3$ points; $1-2$ times per week $=2$ points; $1-2$ times per month $=1$ point; other or no answer $=0$ points), whether parents increased their germ talk (yes $=1$ point; no, other, or no answer $=0$ 
points), the number of days since children had been to school, and the number of days since children had been to a playdate (all predictors mean-centered).

If parents mentioned being in between categories (e.g., 1-2 a week at home, maybe daily at school but not sure), we defaulted to the lower score. The question about new germ-related behaviors was excluded from this analysis because it was written before masks were recommended and the change in that recommendation changed the meaning of this question. Children who had never been to school in person before (e.g., families that were already homeschooling or children who had not yet started to go to school or daycare before the pandemic, $N=8)$ or whose parents did not provide a date estimate $(N=2)$ did not have a value for the school days variable and were not included in the analysis.

We hypothesized three potential associations between these predictors and children's performance in this task. First, we could predict a positive association - more talk or more time since children have been in school could indicate a more disruptive and robust influence of the pandemic on children's lives, which might result in a greater difference in children's performance in high vs. low contact trials. Second, we could predict a negative association perhaps the return to school might signal a new opportunity for children to learn about disease transmission and distancing, which might give children more insight into our task. Third, we could predict no association - what children have learned about COVID-19 or experienced during the pandemic may not generalize to a different novel illness. None of these factors significantly predicted children's performance in this task (see Table 2), the closest being the number of days since children had last been to school, $b=0.25, S E=0.14, t=1.80, p=.075$.

\section{Discussion}


Experiment 4 extended the effects observed in prior experiments - children were most likely to predict a character would get sick if they interacted very closely (i.e., next to each other and touching) compared to interacting at a distance. Again, we observed the most robust effects among older children in our sample. Among 5- and 6-year-olds however, participants differentiated between high and medium interaction trials but not between medium and low interaction trials. From one perspective, this pattern suggests that children might be especially focused on whether characters touched (the difference between high and medium vignettes) rather than proximity (the difference between medium and low vignettes). Alternatively, though this pattern differed from prior experiments, we did not observe a significant effect of experiment (3 vs. 4) or interactions between experiment and other factors, therefore we are hesitant to draw strong conclusions about this result. Comparing across experiments before and during the pandemic, we also observed very little evidence of the effects of the pandemic on children's thinking about disease transmission. There are many ways to quantify this influence, including general pandemic stage (before the pandemic, early, late), number of days since the pandemic started, whether children had anything extra to say about getting sick at the end of the study, parent talk about disease transmission, and disruption to children's lives in terms of being out of school or not interacting with peers. None of these factors were significantly associated with children's performance in our task, a topic we return to in the general discussion.

\section{General Discussion}

The present research suggests that 3- to 6-year-old children can use information about close interactions to make predictions about illness. In Experiment 1, children were more likely to predict that story characters would get sick if they closely interacted with a sick child, compared to situations with more limited interaction. Though children were increasingly likely 
to use information about close interactions with age, even our youngest participants (3- and 4year-old children in Experiment 1) appeared to appreciate the relation between interacting closely with a sick person and disease transmission. Experiment 2 demonstrates that children do not use information about contact when making predictions about any internal state, as they did not predict that characters would become hungry or tired after closely interacting. Finally, in Experiments 3 and 4, children's predictions about disease transmission during the COVID-19 pandemic mirrored the predictions of children tested prior to pandemic. We observed no differences based on whether children were tested before or during the COVID-19 pandemic, indicating that even a highly impactful experience that is discussed frequently at home may not be sufficient to alter young children's basic understanding of disease transmission. These results suggest that considering the role of close interactions may provide children with early insight into the process of disease transmission, although this reasoning may be more fragile among the youngest children.

These findings raise several questions for future research. First, what might facilitate early learning about a complicated and nuanced process such as disease transmission (Christian, 2015; Cohen et al., 1998, 2015)? To highlight the complexity of this phenomenon, perceived social support, including participants' reported frequency of hugging, predicted less severe illness among participants who were all exposed to the common cold virus, despite the close contact between people (Cohen et al., 2015). Educational strategies on that build on children's early theories and intuitions, such as the role of close interactions in disease transmission, may be especially effective for guiding young children's behavior. Children's predictions (rather than their explanations) may be especially important to understand children's existing strategies, as mitigation strategies may be especially important at the prediction stage (i.e., who to avoid being 
close to) rather than the explanation stage (i.e., knowing the outcome of who got sick, how did it happen). Future studies that examine the impact of these educational strategies should collect complementary behavioral data to test whether changes in children's knowledge about germs is related to behaviors that would prevent illness. Although tapping into children's early intuitions may appear to be a simple strategy (e.g., reminding children to stay away from people who appear to be sick), highlighting causal mechanisms may be especially important to complement and build on children's existing theories (e.g., Conrad et al., 2020; Crosby et al., 2019), as well as drive behavioral change. For example, following an intervention that targeted biological causal mechanisms, 8- and 9-year-old children were more likely to wash their hands before preparing a snack (Au et al., 2008; see Gripshover \& Markman, 2013, for a related finding with preschool age children in the domain of nutrition). Simply talking more about disease-prevention behaviors (without targeting causal mechanisms) may not substantially alter children's early intuitions about disease transmission, as suggested by the similarities in children's responses before and during COVID-19 despite the increase in discussion of disease transmission during the pandemic (see also Nguyen \& Rosengren, 2004b). Therefore, providing information about young children's early intuitions about illness and the importance of training around causal mechanisms may be a useful strategy to help parents, educators, and health professionals help children avoid illness and learn about the biological world.

Second, do children's predictions about illness vary across cultures or in contexts (separate from the COVID-19 pandemic) with differing susceptibility to illness? The children tested in the present research live in the United States and are exposed to Western education and perceptions of illness. Biological explanations for illness are shared across many cultures (Nguyen \& Rosengren, 2004a), yet some cross-cultural differences in terms of the causes of 
illness or the severity of contamination are also observed (Hejmadi et al., 2004; Legare et al., 2012; Legare \& Gelman, 2008). For instance, adults in South Africa explain illness using both biological and bewitchment explanations (Legare \& Gelman, 2008), and the endorsement of supernatural explanations for illness increases with age in some cultural contexts (Legare et al., 2012). Children living in India responded more negatively to instances of contamination (e.g., juice that had come in contact with a stranger or a cockroach) and were more likely with age to believe that instances of contamination could not be remedied compared to American children (Hejmadi et al., 2004). Furthermore, urban Chinese children were more likely to prefer vitalist explanations over contagion explanations for illness causation (Nyhof, 2017). Additionally, children may be especially sensitive to the disease risks of their community - children in South Africa demonstrated factual knowledge about AIDS from an early age, yet a deep causal understanding of different illnesses may still unfold over a protracted developmental timeline (Legare et al., 2009).

What have these experiments highlighted about children's experiences and the impact of the COVID-19 pandemic? One takeaway is that school-age children (i.e., 5- and 6-year-olds) have some capacity to understand disease transmission, at least in terms of the likelihood of getting sick from closely interacting with someone (compared to staying further apart and avoiding contact). Nonetheless, we caution against the interpretation that young children can effectively engage in disease-prevention behaviors without support from adults. Most children tested during the pandemic had not yet returned to school or daycare in person at the time they participated in the study and had not had much opportunity to practice behaviors such as maintaining distance from others and keeping masks on for long periods of time. Frequent reminders, routines, and support from adults may be necessary during this time and potential 
future pandemics, especially if the youngest children have trouble generalizing from one illness to another. Children may have learned a lot about COVID-19 during this period, but may not generalize that knowledge to novel illnesses (which in some ways is challenging to guide behavior but in other ways is rational, as different illnesses require different mitigation strategies). To provide an anecdotal example, one 4-year-old child who responded somewhat randomly in this task (high-low difference $=0.50$, with 0 indicating no difference between high and low interaction trials and 3 being the maximum possible difference) mentioned "don't get close to people when you're not wearing masks" in their open-ended response.

Another takeaway regarding the impact of the pandemic is that quantifying impact is challenging. As we highlight in Experiments 3-4, we did not observe evidence of effects of the pandemic in terms of parent talk about illness or days since children had been to school or a playdate on their performance in this task. This pattern potentially suggests that this aspect of children's reasoning may be especially invariant to contextual influence. Another explanation could be that the pandemic is affecting children's thinking about disease transmission in more complex ways than we measured in this study or could have planned at the start of the pandemic. For example, children's experience with school during the pandemic is more complex than just the number of days since children had been to school. Some schools have opened and closed several times; different schools may have different requirements for behavior at school. Some children were going to school in person for the first time, whereas others had experience going to school before and during COVID, both highlighting the difference in those experiences but also confounded with child age (i.e., the oldest children who we would expect to do best at this task were also the most likely to have experienced this change in school environment). Future research is needed to fully understand how to quantify the impact of COVID-19 on children's 
daily experiences and the potential influence of that impact on children's reasoning about disease transmission.

Finally, the COVID-19 pandemic has raised many questions about the representativeness of findings obtained during this period (see Lourenco \& Tasimi, 2020). Although access to the Internet and devices such as laptops, tablets, and smartphones is widespread in the U.S. (Pew Research Center, 2019), a digital divide is nonetheless observed, with families in rural and impoverished communities having less access than families in more urbanized or affluent communities (van Dijk, 2020). Moreover, the videoconference method may be especially challenging for the youngest participants (such as 3- and 4-year-olds), who may have less experience with these methods than school-age children. Although we did not observe effects of experiment (i.e., before vs. during COVID-19), we also did not observe an effect of interaction type among 3- and 4-year-olds tested during the pandemic. This could represent the fragility of young children's reasoning about disease transmission, general challenges of conducting studies with young children by videoconference, or both. Separate from the research questions of this study, the transition to conducting studies online and the discussions in our parent interviews highlight the disruptiveness of the pandemic to every aspect of the lives of children and families. These conversations aligned with other studies highlighting the impact of COVID-19 on the psychological well-being of children and parents (Gassman-Pines et al., 2020) and showcased many highs and lows. Some children seemed excited to talk to us and showed us their favorite toy; a few parents reported that their children were thriving at home or that they were enjoying the opportunity to see their children learn. On the other hand, several parents shared difficult challenges. One parent discussed speaking to their child about keeping those suffering from COVID-19 in their prayers. Another shared that their children were having nightmares about 
COVID-19. Another discussed the high strain on mothers and inequities in caregiving during this period (see Malisch et al., 2020; Viglione, 2020, for discussions of the impact of COVID-19 on women in academia). Future research will be critical to fully understand how this pandemic has impacted children and families beyond these anecdotal, yet powerful, examples. 


\section{References}

American Academy of Pediatrics. (2020). Research Highlights: COVID-19 and Pediatric Populations. American Academy of Pediatrics. https://www.aappublications.org/sites/default/files/additional_assets/aap_files/AAPSum mary-COVID-19-Pediatric-Research-Highlights-7.14.20.pdf

Au, T. K., Chan, C. K. K., Chan, T., Cheung, M. W. L., Ho, J. Y. S., \& Ip, G. W. M. (2008). Folkbiology meets microbiology: A study of conceptual and behavioral change. Cognitive Psychology, 57(1), 1-19. https://doi.org/10.1016/j.cogpsych.2008.03.002

Au, T. K., Sidle, A. L., \& Rollins, K. B. (1993). Developing an intuitive understanding of conservation and contamination: Invisible particles as a plausible mechanism. Developmental Psychology, 29(2), 286-299. https://doi.org/10.1037/0012-1649.29.2.286

Blacker, K.-A., \& LoBue, V. (2016). Behavioral avoidance of contagion in childhood. Journal of Experimental Child Psychology, 143, 162-170. https://doi.org/10.1016/j.jecp.2015.09.033

Bonoti, F., Christidou, V., \& Spyrou, G. M. (2019). 'A smile stands for health and a bed for illness': Graphic cues in children's drawings. Health Education Journal, 78(7), 728-742. https://doi.org/10.1177/0017896919835581

Carey, S. (1985). Conceptual change in childhood. MIT Press.

Centers for Disease Control and Prevention. (2016a). Common Cold. https://www.cdc.gov/dotw/common-cold/

Centers for Disease Control and Prevention. (2016b). Influenza (Flu): Information for Schools \& Childcare Providers. https://www.cdc.gov/flu/school/ 
Christian, L. M. (2015). Stress and immune function during pregnancy. Current Directions in Psychological Science, 24(1), 3-9. https://doi.org/10.1177/0963721414550704

Cohen, S., Frank, E., Doyle, W. J., Skoner, D. P., Rabin, B. S., \& Gwaltney Jr, J. M. (1998). Types of stressors that increase susceptibility to the common cold in healthy adults. Health Psychology, 17(3), 214-223. https://doi.org/10.1037/0278-6133.17.3.214

Cohen, S., Janicki-Deverts, D., Turner, R. B., \& Doyle, W. J. (2015). Does hugging provide stress-buffering social support? A study of susceptibility to upper respiratory infection and illness. Psychological Science, 26(2), 135-147. https://doi.org/10.1177/0956797614559284

Conrad, M., Kim, E., Blacker, K.-A., Walden, Z., \& LoBue, V. (2020). Using storybooks to teach children about illness transmission and promote adaptive health behavior - A pilot study. Frontiers in Psychology, 11, 942. https://doi.org/10.3389/fpsyg.2020.00942

Crosby, S., Laird, K., \& Younie, S. (2019). Interactive health-hygiene education for early years: The creation and evaluation of learning resources to improve understanding of handwashing practice. International Journal of Early Years Education, 27(4), 374-390. https://doi.org/10.1080/09669760.2019.1628010

DeJesus, J. M., Shutts, K., \& Kinzler, K. D. (2015). Eww she sneezed! Contamination context affects children's food preferences and consumption. Appetite, 87, 303-309. https://doi.org/10.1016/j.appet.2014.12.222

Fallon, A. E., Rozin, P., \& Pliner, P. (1984). The child's conception of food: The development of food rejections with special reference to disgust and contamination sensitivity. Child Development, 55(2), 566-575. https://doi.org/10.2307/1129968 
Gassman-Pines, A., Ananat, E. O., \& Fitz-Henley, J. (2020). COVID-19 and Parent-Child Psychological Well-being. Pediatrics, 146(4), e2020007294. https://doi.org/10.1542/peds.2020-007294

Gelman, S. A. (2003). The essential child: Origins of essentialism in everyday thought. Oxford University Press.

Gelman, S. A., \& Wellman, H. M. (1991). Insides and essences: Early understandings of the non-obvious. Cognition, 38(3), 213-244. https://doi.org/10.1016/0010-0277(91)90007-Q

Gopnik, A. (2012). Scientific thinking in young children: Theoretical advances, empirical research, and policy implications. Science, 337(6102), 1623-1627. https://doi.org/10.1126/science.1223416

Gopnik, A., Glymour, C., Sobel, D. M., Schulz, L. E., Kushnir, T., \& Danks, D. (2004). A theory of causal learning in children: Causal maps and Bayes nets. Psychological Review, 111(1), 3-32. https://doi.org/10.1037/0033-295X.111.1.3

Gripshover, S. J., \& Markman, E. M. (2013). Teaching young children a theory of nutrition conceptual change and the potential for increased vegetable consumption. Psychological Science, 24(8), 1541-1553. https://doi.org/10.1177/0956797612474827

Hatano, G., \& Inagaki, K. (2013). Young children's thinking about biological world. Psychology Press.

Hejmadi, A., Rozin, P., \& Siegal, M. (2004). Once in contact, always in contact: Contagious essence and conceptions of purification in American and Hindu Indian children. Developmental Psychology, 40(4), 467-476. https://doi.org/10.1037/0012-1649.40.4.467 
Jarosz, A. F., \& Wiley, J. (2014). What Are the Odds? A Practical Guide to Computing and Reporting Bayes Factors. The Journal of Problem Solving, 7(1). https://doi.org/10.7771/1932-6246.1167

Johansson, M. A., Quandelacy, T. M., Kada, S., Prasad, P. V., Steele, M., Brooks, J. T., Slayton, R. B., Biggerstaff, M., \& Butler, J. C. (2021). SARS-CoV-2 transmission from people without COVID-19 symptoms. JAMA Network Open, 4(1), e2035057. https://doi.org/10.1001/jamanetworkopen.2020.35057

Kalish, C. W. (1996a). Causes and symptoms in preschoolers' conceptions of illness. Child Development, 67(4), 1647-1670. https://doi.org/10.1111/j.1467-8624.1996.tb01819.x

Kalish, C. W. (1996b). Preschoolers' understanding of germs as invisible mechanisms. Cognitive Development, 11(1), 83-106. https://doi.org/10.1016/S0885-2014(96)90029-5

Kalish, C. W. (1997). Preschoolers' understanding of mental and bodily reactions to contamination: What you don't know can hurt you, but cannot sadden you. Developmental Psychology, 33(1), 79-91. https://doi.org/10.1037/0012-1649.33.1.79

Kalish, C. W. (1998). Young children's predictions of illness: Failure to recognize probabilistic causation. Developmental Psychology, 34(5), 1046-1058. https://doi.org/10.1037/00121649.34.5.1046

Kloepfer, K. M., Lee, W. M., Pappas, T. E., Kang, T. J., Vrtis, R. F., Evans, M. D., Gangnon, R. E., Bochkov, Y. A., Jackson, D. J., Lemanske Jr, R. F., \& Gern, J. E. (2014). Detection of pathogenic bacteria during rhinovirus infection is associated with increased respiratory symptoms and asthma exacerbations. Journal of Allergy and Clinical Immunology, 133(5), 1301-1307.e3. https://doi.org/10.1016/j.jaci.2014.02.030 
Legare, C. H., Evans, E. M., Rosengren, K. S., \& Harris, P. L. (2012). The coexistence of natural and supernatural explanations across cultures and development. Child Development, 83(3), 779-793. https://doi.org/10.1111/j.1467-8624.2012.01743.x

Legare, C. H., \& Gelman, S. A. (2008). Bewitchment, biology, or both: The co-existence of natural and supernatural explanatory frameworks across development. Cognitive Science, 32(4), 607-642. https://doi.org/10.1080/03640210802066766

Legare, C. H., Wellman, H. M., \& Gelman, S. A. (2009). Evidence for an explanation advantage in naïve biological reasoning. Cognitive Psychology, 58(2), 177-194. https://doi.org/10.1016/j.cogpsych.2008.06.002

Lourenco, S. F., \& Tasimi, A. (2020). No participant left behind: Conducting science during COVID-19. Trends in Cognitive Sciences, 24(8), 583-584. https://doi.org/10.1016/j.tics.2020.05.003

Malisch, J. L., Harris, B. N., Sherrer, S. M., Lewis, K. A., Shepherd, S. L., McCarthy, P. C., Spott, J. L., Karam, E. P., Moustaid-Moussa, N., Calarco, J. M., Ramalingam, L., Talley, A. E., Cañas-Carrell, J. E., Ardon-Dryer, K., Weiser, D. A., Bernal, X. E., \& Deitloff, J. (2020). Opinion: In the wake of COVID-19, academia needs new solutions to ensure gender equity. Proceedings of the National Academy of Sciences, 117(27), 15378. https://doi.org/10.1073/pnas.2010636117

Margett-Jordan, T., Falcon, R. G., \& Witherington, D. C. (2017). The development of preschoolers' living kinds concept: A longitudinal study. Child Development, 88(4), 1350-1367. https://doi.org/10.1111/cdev.12709

Muñoz Marco, V., Cano Martil, S., Munoz Sastre, M. T., Sorum, P. C., \& Mullet, E. (2017). Mapping children's and adolescents' judgment rules for assessing the risk of disease 
transmission from sick friends: Contagion. Infant and Child Development, 26(5), e2014. https://doi.org/10.1002/icd.2014

Nguyen, S. P., \& Rosengren, K. S. (2004a). Causal reasoning about illness: A comparison between European-and Vietnamese-American children. Journal of Cognition and Culture, 4(1), 51-78. https://doi.org/10.1163/156853704323074750

Nguyen, S. P., \& Rosengren, K. S. (2004b). Parental reports of children’s biological knowledgeand misconceptions. International Journal of Behavioral Development, 28(5), 411-420. https://doi.org/10.1080/01650250444000108

Nyhof, M. (2017). Intuitive foundations of conceptions of vitality: The case of Chinese children's understanding of illness causation. In R. G. Hornbeck, J. L. Barrett, \& M. Kang (Eds.), Religious Cognition in China (Vol. 2, pp. 139-149). Springer International Publishing. https://doi.org/10.1007/978-3-319-62954-4_9

Pew Research Center. (2019). Demographics of Internet and Home Broadband Usage in the United States. Internet/Broadband Fact Sheet. https://www.pewresearch.org/internet/factsheet/internet-broadband/

Raman, L., \& Gelman, S. A. (2007). Children's recognition of time in the causes and cures of physical and emotional reactions to illnesses and injuries. British Journal of Psychology, 98(3), 389-410. https://doi.org/10.1348/000712606X147790

Rolfes, M., Foppa, I., Garg, S., Flannery, B., Brammer, L., Singleton, J., Burns, E., Jernigan, D., Reed, C., Olsen, S., \& Bresee, J. (2016). Estimated Influenza Illnesses, Medical Visits, Hospitalizations, and Deaths Averted by Vaccination in the United States. https://www.cdc.gov/flu/about/disease/2015-16.htm 
Rozin, P., \& Fallon, A. E. (1987). A perspective on disgust. Psychological Review, 94(1), $23-41$. https://doi.org/10.1037/0033-295X.94.1.23

Siegal, M. (1988). Children's knowledge of contagion and contamination as causes of illness. Child Development, 59(5), 1353-1359. https://doi.org/10.2307/1130497

Slaughter, V., \& Lyons, M. (2003). Learning about life and death in early childhood. Cognitive Psychology, 46(1), 1-30. https://doi.org/10.1016/S0010-0285(02)00504-2

Sobel, D. M., Yoachim, C. M., Gopnik, A., Meltzoff, A. N., \& Blumenthal, E. J. (2007). The blicket within: Preschoolers' inferences about insides and causes. Journal of Cognition and Development, 8(2), 159-182. https://doi.org/10.1080/15248370701202356

Solomon, G. E. A., \& Cassimatis, N. L. (1999). On facts and conceptual systems: Young children's integration of their understandings of germs and contagion. Developmental Psychology, 35(1), 113-126. https://doi.org/10.1037/0012-1649.35.1.113

Springer, K., \& Ruckel, J. (1992). Early beliefs about the cause of illness: Evidence against immanent justice. Cognitive Development, 7(4), 429-443. https://doi.org/10.1016/08852014(92)80002-W

Stevenson, R. J., Oaten, M. J., Case, T. I., Repacholi, B. M., \& Wagland, P. (2010). Children's response to adult disgust elicitors: Development and acquisition. Developmental Psychology, 46(1), 165-177. https://doi.org/10.1037/a0016692

van Dijk, J. (2020). The Digital Divide. John Wiley \& Sons, Inc.

Viglione, G. (2020). Are women publishing less during the pandemic? Here's what the data say. Nature, 581, 365-366. https://doi.org/10.1038/d41586-020-01294-9 
Xu, F., \& Tenenbaum, J. B. (2007). Sensitivity to sampling in Bayesian word learning. Developmental Science, 10(3), 288-297. https://doi.org/10.1111/j.14677687.2007.00590.x 
Appendix

\section{Study Vignettes}

\section{Experiments 1 \& 3: Children's Predictions About Disease Transmission}

Low interaction: This is Jacob. He is sick with zibroff. Even though he wasn't feeling well, he wanted to go to his playdate with Michael. Jacob and Michael play across the room from each other and don't touch each other at all. After playing with Jacob, do you think Michael gets sick with zibroff or does not get sick with zibroff?

Medium interaction: This is Hannah. She is sick with febrel. Even though she wasn't feeling well, she wanted to go to her playdate with Natalie. Hannah and Natalie play nearby each other and only touch each other a little bit. After playing with Hannah, do you think Natalie gets sick with febrel or does not get sick with febrel?

High interaction: This is Andrea. She is sick with kraylar. Even though she wasn't feeling well, she wanted to go to her playdate with Rachel. Andrea and Rachel play right next to each other and touch each other a lot. After playing with Andrea, do you think Rachel gets sick with kraylar or does not get sick with kraylar?

\section{Experiment 2: Children's Predictions About Other Internal states}

Hungry trial: This is David. He didn't have time to eat breakfast today. Even though he was hungry, he wanted to go to his playdate with Ryan. David and Ryan play nearby each other and only touch each other a little bit. After playing with David, do you think Ryan gets hungry or does not get hungry?

Tired trial: This is Grace. She didn't have time to take a nap today. Even though she was tired she wanted to go to her playdate with Lauren. Grace and Lauren play across the room from each other and don't touch each other at all. After playing with Grace, do you think Lauren gets tired or does not get tired?

\section{Experiment 4: Extending Children's Predictions About Illness During COVID-19}

Medium interaction: This is Hannah. She is sick with febrel. Even though she wasn't feeling well, she wanted to go to her playdate with Natalie. Hannah and Natalie play right next to each other and don't touch each other at all (low and high interaction identical to Experiments 1 \& 3 ).

\section{COVID-19 Parent Questionnaire}

How often do you talk to your child about germs? (response options: daily, 1-2 times per week, 1-2 times per month, something else).

What kinds of things have you been talking about? (open-ended)

Has the amount you speak with them about germs increased since COVID-19? (response options: yes, no, other) 
Lately, have you explicitly taught your child any new germ-related behaviors? (response options: yes, no, other). This question was written at the start of the pandemic (not knowing how things would unfold), so when asked later in the pandemic (November 2020), this was clarified as "since the pandemic started."

(if yes) What kinds of things have you been talking about? (open-ended)

What explanations are you giving your child about the current stay-at-home situation? (openended)

If you can remember, what was the last date that your child attended school, daycare, or a similar activity?

If you can remember, what was the last date that your child had a playdate with a friend?

Is there anything else you'd like to tell us about how the current health crisis is impacting your or your child's daily activities? (open-ended) 
Figure 1
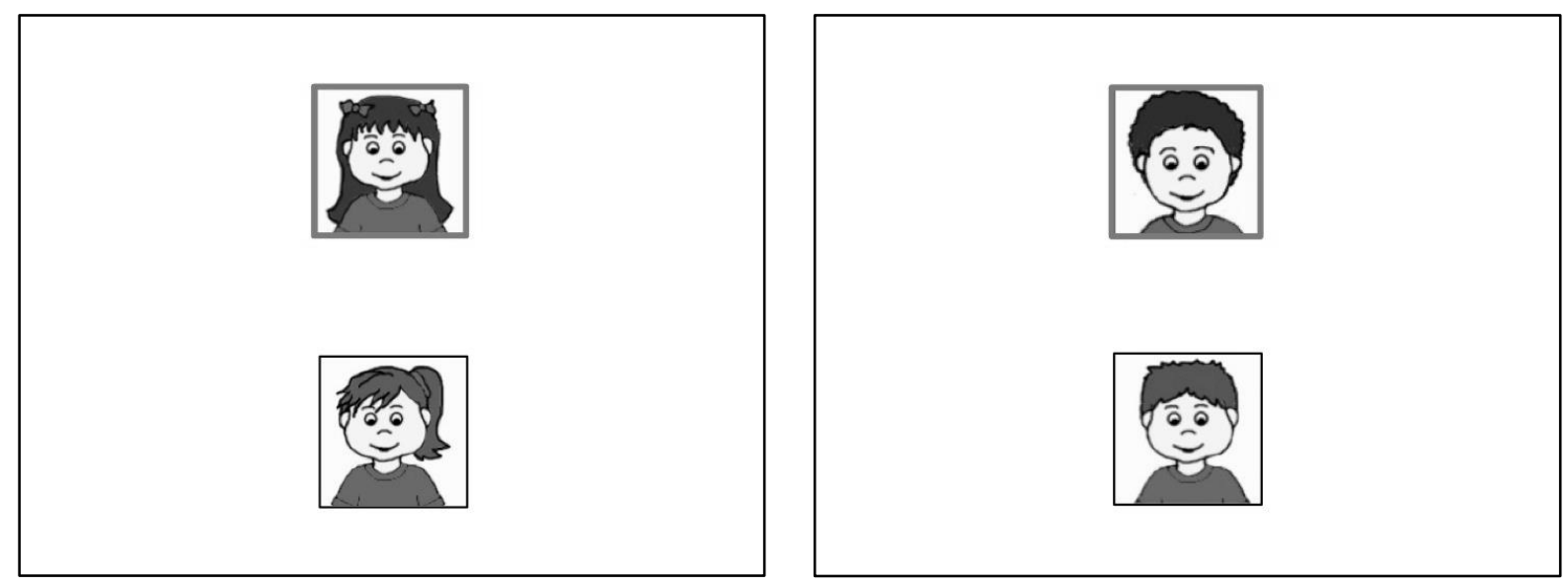

Figure 1. Exemplars of test trials. The top character was described as sick (Experiment 1, 3, 4) or hungry/tired (Experiment 2). Children were asked to predict whether the bottom character would get sick (Experiment 1, 3, 4) or hungry/tired (Experiment 2). 
Figure 2

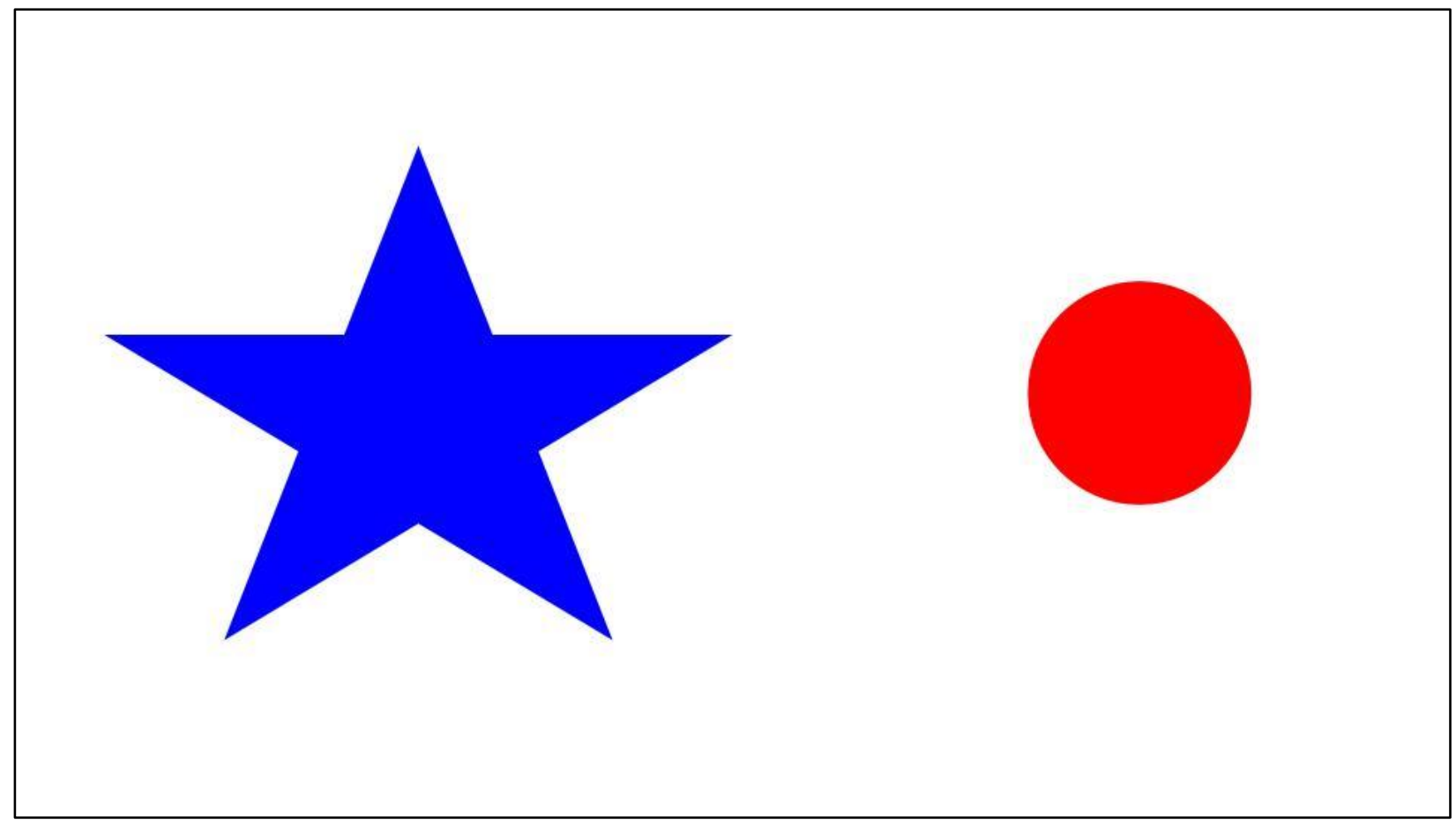

Figure 2. Shapes used for calibration check in Experiment 3-4. 
Figure 3

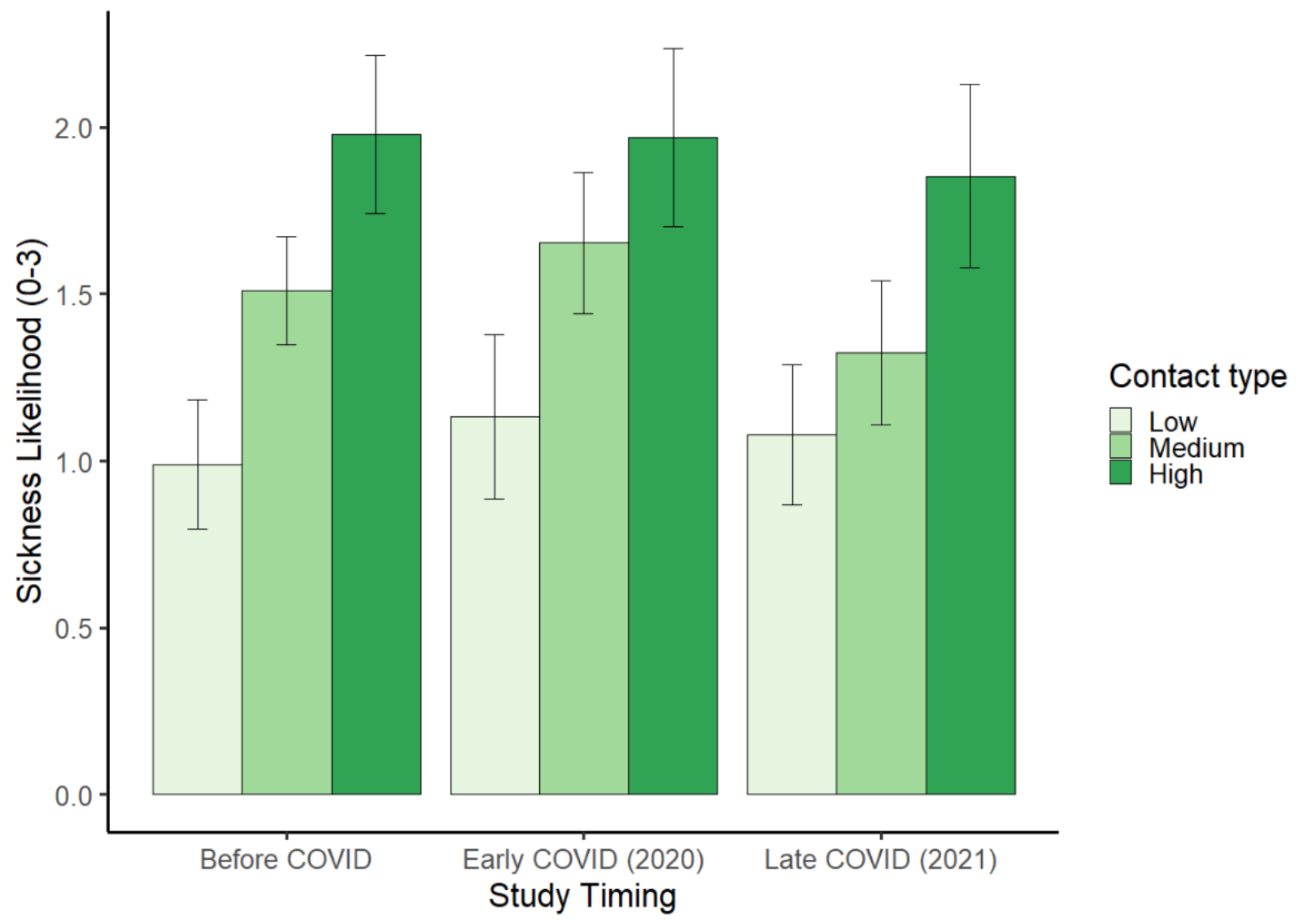

Figure 3. Results from before the pandemic (left), early in the pandemic (center) and later in the pandemic (right), including data from Experiments 1, 3, and 4. Error bars represent 95\% confidence intervals. 
Table 1

Descriptive data from parent interview in Experiments 3 and 4 (during COVID). Data provided are means (with $95 \%$ confidence intervals) or $N(\%)$ depending on the question. In Experiment 4 , one parent declined to complete the parent interview.

\begin{tabular}{|c|c|c|c|}
\hline & $\begin{array}{c}\text { Experiment } 3 \\
(N=48)\end{array}$ & $\begin{array}{c}\text { Experiment } 4 \\
(N=51)\end{array}$ & $\begin{array}{c}\text { Total } \\
(N=99) \\
\end{array}$ \\
\hline Days in pandemic & 207 days $(183,232)$ & 335 days $(313,327)$ & 273 days $(239,275)$ \\
\hline $\begin{array}{l}\text { Child open-ended response } \\
\text { (illness-relevant) }\end{array}$ & $11(23 \%)$ & $15(29 \%)$ & $26(26 \%)$ \\
\hline \multicolumn{4}{|l|}{ Germ talk frequency } \\
\hline Daily & $27(56 \%)$ & $30(59 \%)$ & $57(58 \%)$ \\
\hline 1-2 times per week & $15(31 \%)$ & $20(39 \%)$ & $35(35 \%)$ \\
\hline 1-2 times per month & $1(2 \%)$ & $1(2 \%)$ & $2(2 \%)$ \\
\hline Something else & $5(8 \%)$ & $0(0 \%)$ & $5(5 \%)$ \\
\hline \multicolumn{4}{|l|}{ Increase in germ talk } \\
\hline Yes & $40(83 \%)$ & $45(88 \%)$ & $85(86 \%)$ \\
\hline No & $6(13 \%)$ & $1(2 \%)$ & $7(7 \%)$ \\
\hline Other & $2(4 \%)$ & $5(10 \%)$ & $7(7 \%)$ \\
\hline \multicolumn{4}{|l|}{ New germ-related behaviors } \\
\hline Yes & $35(73 \%)$ & $46(90 \%)$ & $81(82 \%)$ \\
\hline No & $10(21 \%)$ & $3(6 \%)$ & $13(13 \%)$ \\
\hline Other & $3(6 \%)$ & $2(4 \%)$ & $5(5 \%)$ \\
\hline Days since school/daycare & 124 days $(90,158)$ & 112 days $(64,160)$ & 118 days $(89,147)$ \\
\hline Been to school in person? & $16(33 \%)$ & $33(65 \%)$ & $49(49 \%)$ \\
\hline Days since playdate & 56 days $(30,81)$ & 80 days $(43,118)$ & 68 days $(46,91)$ \\
\hline
\end{tabular}


Table 2

Regression analysis of individual differences in children's pandemic experience.

\begin{tabular}{lrrrr}
\hline & Estimate & \multicolumn{1}{c}{ SE } & \multicolumn{1}{c}{$\boldsymbol{c}$} & $\boldsymbol{p}$-value \\
\hline (Intercept) & 0.87 & 0.13 & 6.49 & $<.001$ \\
Child open-ended response (illness-relevant) & 0.09 & 0.13 & 0.69 & .494 \\
Germ talk frequency & -0.06 & 0.15 & -0.37 & .714 \\
Increase in germ talk & 0.17 & 0.14 & 1.21 & .230 \\
Days since school/daycare & 0.25 & 0.14 & 1.80 & .075 \\
Days since playdate & -0.06 & 0.14 & -0.43 & .668 \\
\hline
\end{tabular}

